\title{
Potent bronchodilation and reduced stiffness by relaxant stimuli under dynamic conditions
}

\author{
T.K. Ansell, P.K. McFawn, P.B. Noble, A.R. West, L. Fernandes and H.W. Mitchell
}

ABSTRACT: Airway relaxation in response to isoprenaline, sodium nitroprusside (SNP) and electrical field stimulation (EFS) was compared under static and dynamic conditions. The capacity of relaxants to reduce airway stiffness and, thus, potentially contribute to bronchodilation was also investigated.

Relaxation responses were recorded in fluid filled bronchial segments from pigs under static conditions and during volume oscillations simulating tidal and twice tidal manoeuvres. Bronchodilation was assessed from the reduction in carbachol-induced lumen pressure, at isovolume points in pressure cycles produced by volume oscillation, and stiffness was assessed from cycle amplitudes.

Under static conditions, all three inhibitory stimuli produced partial relaxation of the carbacholinduced contraction. Volume oscillation alone also reduced the contraction in an amplitudedependent manner. However, maximum relaxation was observed when isoprenaline or SNP were combined with volume oscillation, virtually abolishing contraction at the highest drug concentrations. The proportional effects of isoprenaline and EFS were not different under static or oscillating conditions, whereas relaxation to SNP was slightly greater in oscillating airways. All three inhibitory stimuli also strongly reduced carbachol-induced airway stiffening.

The current authors conclude that bronchoconstriction is strongly suppressed by combining the inhibitory stimulation of airway smooth muscle with cyclical mechanical strains. The capacity of airway smooth muscle relaxants to also reduce stiffness may further contribute to bronchodilation.

KEYWORDS: Airway smooth muscle, airway stiffness, bronchial responsiveness, bronchodilation, nitric oxide

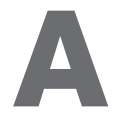
irway calibre is regulated by neural, pharmacological and mechanical factors providing either excitatory or inhibitory input to airway smooth muscle (ASM). In recent years, the importance of the dynamic mechanical environment of the lung during normal respiration has also become increasingly recognised, particularly the loss of ASM active force produced by a range of contractile agents in response to the dynamic strain of tidal and deep breathing [1-4]. The precise mechanism accounting for the straininduced regulation of force is not certain but likely includes alterations in cross-bridge cycling and/or cytoskeletal and contractile protein plasticity $[3,5]$. Thus, ASM tone and airway calibre is regulated by summed and possibly interlacing effects of the various signalling pathways evoked by contractile agonist-induced airway activation and by downstream contractile regulation of ASM through the dynamic mechanical environment of the lung.
Inhibitory inputs to ASM are less well understood in this mechanical environment. As noted by Fust and STEPHENS [6], the capacity of ASM to relax could be just as important to airway physiology and to hyperresponsiveness as its capacity to contract. To the present authors' knowledge, there is only one report comparing pharmacologically evoked relaxation under static isoprenaline and strain each reduced force in isolated ASM; however, there was no evidence that the pharmacological and mechanical interventions had substantial synergistic effects [4]. It is not currently known how oscillatory strain interacts with other inhibitory signalling pathways. Inhibitory input to ASM in vivo typically involves both pharmacological (e.g. $\beta_{2}$ adrenoreceptor agonists) and neural input, the latter principally via nitrergic neurotransmission [7-9]. Nitric oxide from neural and non-neural sources and oscillatory conditions [4]. In that study,

\section{AFFILIATIONS}

Discipline of Physiology, School of Biomedical, Biomolecular and Chemical Sciences and Discipline of Pharmacology, School of Medicine and Pharmacology, University of Western Australia, Perth, Australia.

\section{CORRESPONDENCE}

H.W. Mitchell

Discipline of Physiology, School of

Biomedical, Biomolecular and Chemical Sciences

University of Western Australia 35 Stirling Hwy

Perth

6009

Australia

Fax: 61864881025

E-mail: mitchell@

cyllene.uwa.edu.au

Received:

July 312008

Accepted after revision:

October 172008

SUPPORT STATEMENT

The salary for $P$. Noble was provided by the Raine Medical Research

Foundation of Western Australia (Nedlands, Australia). The present work was supported by a project grant from the National Health and Medical Research Council of Australia (Canberra, Australia).

STATEMENT OF INTEREST

None declared. 
is also considered to play a role in inflammatory disease, including pro-inflammatory and ASM relaxant effects [10]. Given the importance of dynamic conditions to lung function, the relaxant response of ASM in the presence of inhibitory stimulation and cyclical strain together is clearly relevant to the maintenance of normal airway calibre and possibly excessive airway narrowing in disease.

In the present study, the effects of cyclical strain on the efficacy of a number of relaxant stimuli, namely nerve-evoked relaxation, a nitric oxide donor (sodium nitroprusside (SNP)) and isoprenaline were determined. The different cellular signalling pathways for SNP, isoprenaline and nerve stimulation may also provide information about possible points of intersection between the processes responsible for the regulation of ASM contraction by mechanical strain and the regulation of contraction by pharmacological and neural stimuli. Studies were carried out in an intact airway preparation that models the in vivo situation where mechanical strain is produced by cyclical changes in transmural pressure, which stresses then strains ASM within the wall. This approach is preferred to isolated ASM studies, since the amount of and kinetics of ASM strain in the wall depends on the stiffness of the various structures that make up the multilayered airway wall. In other words, as well as producing bronchodilation as a direct result of ASM relaxation, inhibitory stimuli may also reduce the stiffness of the airway, which could favour bronchodilation as a result of greater ASM strain to mechanical oscillation. Since the effects of bronchodilators on the stiffness of airways have not been previously documented, the current authors also assessed pressure-volume properties of airway segments following relaxation to neural and pharmacological stimulation. The present findings further support the importance of the dynamic conditions in the lung and of airway stiffness in regulating airway calibre.

\section{METHODS}

\section{Tissue preparation}

All animal experiments conformed to the institutional ethics and animal care unit regulations (University of Western Australia, Animal Ethics Committee, Perth, Australia). Male White Landrace pigs $\sim 25 \mathrm{~kg}$ were initially sedated with tiletaminezolazepam $\left(4.4 \mathrm{mg} \cdot \mathrm{kg}^{-1}\right.$ i.m.) and xylazine $\left(2.2 \mathrm{mg} \cdot \mathrm{kg}^{-1}\right.$ i.m.) and then exsanguinated under sodium pentobarbitone anaesthesia (30 mg. $\mathrm{kg}^{-1}$ i.v.). The lungs were removed and transported on ice to the laboratory.

Segments of bronchi were dissected from the main stem bronchus of the left lower lobe. All side branches were ligated and a 20-mm long segment of bronchus $\sim 2 \mathrm{~mm}$ in internal diameter was cannulated at both ends for mounting in an organ chamber. After cannulation, the bronchus was mounted horizontally in an organ bath containing gassed $\left(95 \% \mathrm{O}_{2}\right.$ and $5 \% \mathrm{CO}_{2}$ ) Krebs solution (121 mM NaCl, $5.4 \mathrm{mM} \mathrm{KCl}, 1.2 \mathrm{mM}$ $\mathrm{MgSO}_{4}, 25 \mathrm{mM} \mathrm{NaHCO}_{3}, 5.0 \mathrm{mM}$ sodium morpholinopropane sulfonic acid, $11.5 \mathrm{mM}$ glucose and $2.5 \mathrm{mM} \mathrm{CaCl}{ }_{2}$ ) at $\mathrm{pH} 7.3$ maintained at $37^{\circ} \mathrm{C}$. The lumen of the bronchus was connected via a three way tap to a reservoir filled with Krebs solution while the opposite end of the segment was connected to a servo-controlled $1 \mathrm{~mL}$ syringe. Opening the taps allowed the lumen to be flushed with Krebs solution from the reservoir. All connections were made with stiff polyethylene tubing to minimise system compliance and were leak free. The length of the segment was stretched to $105 \%$ of its length in the fully deflated lung, shown previously to approximate the length at functional residual capacity [11]. Initially, intraluminal (and therefore transmural) pressure was set to $5 \mathrm{cmH}_{2} \mathrm{O}(0.49 \mathrm{kPa})$ using the reservoir filled with Krebs solution and intraluminal pressure was measured by a calibrated transducer (Gould, model P23ID) and a PowerLab data-acquisition system (ADInstruments, Bella Vista, Australia).

After dissection and mounting, segments were allowed to equilibrate to organ bath conditions for $1 \mathrm{~h}$ under a static passive pressure of $5 \mathrm{cmH}_{2} \mathrm{O}(0.49 \mathrm{kPa})$. Viability of the tissue was confirmed through stimulation with acetylcholine ( $\mathrm{ACh}$; $\left.10^{-4} \mathrm{M}\right)$. Closure of a tap inserted between the segment and the reservoir allowed contraction of the bronchi (e.g. to ACh) to be measured as a rise in intraluminal pressure, defined as active pressure.

\section{Volume oscillation}

Cyclical oscillations in intraluminal volume, and therefore ASM circumference, were produced using a servo-controlled syringe. The syringe plunger was driven by a direct-current motor (model JDTH-2250-FX-1C; Litton Clifton Precision, Clifton Heights, PA, USA) via a BioPWM sequential motor controller (model V1.0) and custom-designed software (S. De Catania (c) 2005). Feedback control on the motor controller was provided through a linear displacement potentiometer attached to the syringe plunger. This servo-controlled system allowed amplitude and frequency of sinusoidal changes in volume to be controlled. Except where stated, each bronchial segment was studied under static conditions and under volume oscillations to simulate tidal breaths and twice tidal breaths. To simulate tidal breaths, the amplitude of sinusoidal volume oscillations was chosen so that under relaxed conditions they produced a pressure swing from $5-10 \mathrm{cmH}_{2} \mathrm{O}$ $\left(0.49-0.98 \mathrm{kPa}\right.$; i.e. a trough at $5 \mathrm{cmH}_{2} \mathrm{O}$ and a peak at $10 \mathrm{cmH}_{2} \mathrm{O}$ giving a tidal change of $5 \mathrm{cmH}_{2} \mathrm{O}$ ) at a frequency of $0.25 \mathrm{~Hz}$. In a previous study, tidal volume oscillations were shown to produce an $\sim 4 \%$ increase in ASM circumference [12]. For twice tidal breaths, the volume of oscillation was double that used for tidal oscillation.

\section{Experimental protocol}

Three separate groups of bronchi were used to determine the relaxant responses to nerve stimulation, a nitric oxide donor (SNP) and isoprenaline under static and oscillating conditions.

The protocol for recording inhibitory electrical field stimulation (EFS) responses in pig airways was taken from KANNAN and JoHNSON [8] and ConNellan and Mitchell [9]. Briefly, bronchi were exposed to a low concentration $\left(10^{-7} \mathrm{M}\right)$ of atropine for $20 \mathrm{~min}$, which blocks contributing effects from cholinergic nerves on the EFS-induced response. After thorough washout and under either the oscillating or static conditions, airways were contracted with carbachol. To standardise active pressure, a concentration of carbachol was used $\left(10^{-5} \mathrm{M}\right)$ that produced $\sim 50 \%$ of the maximum contraction seen in nonatropinised preparations under static conditions. EFS trains of $30 \mathrm{~s}$ (299 mA, $20 \mathrm{~Hz}$ and $2 \mathrm{~ms}$ pulse width) were applied to the carbachol-contracted bronchi through platinum electrodes surrounding the segment, using a Grass Medical Instrument 
stimulator (model S44; Quincy, MA, USA) and Ugo Basile pulse booster (model T3165; Comerio, VA, USA). In these atropinised preparations, EFS produced a relaxant response, i.e. a decrease in active lumen pressure. EFS-induced relaxations were recorded under static, tidal and twice tidal conditions, in a randomised sequence.

A separate protocol was used in two further groups of bronchi to determine relaxant responses to SNP and isoprenaline. Airway preparations were pre-contracted with carbachol, under static or oscillating conditions. To provide a similar amount of active pressure used in the EFS experiment, a concentration of carbachol $\left(10^{-6} \mathrm{M}\right)$ was used that produced $\sim 50 \%$ of its maximum response under static conditions. This concentration of carbachol was less than the EFS protocol, because the airways had not been exposed to atropine. Once the static or oscillating active pressure was stable, SNP $\left(3 \times 10^{-6}, 10^{-5}\right.$ and $\left.3 \times 10^{-5} \mathrm{M}\right)$ or isoprenaline $\left(10^{-7}, 10^{-6}\right.$ and $\left.10^{-5} \mathrm{M}\right)$ was added. Each concentration was added cumulatively once response to the preceding concentration had stabilised ( $\sim 5 \mathrm{~min}$ per concentration). An example of an isoprenaline-induced relaxation response is shown in figure 1. Separate runs were made under static, tidal or twice tidal conditions, in a randomised sequence.

\section{Analysis and statistics}

Passive pressure was defined as the pressure imposed on the bronchus by the apparatus pressure head and active pressure was defined as the change in pressure at the same volume produced by ASM contraction. Under oscillatory conditions, active pressure was measured at the troughs in the pressure cycles since these isovolume points enable direct comparison between static and oscillatory conditions. To investigate possible interactions between oscillation and EFS, SNP or isoprenaline, evoked relaxation was normalised against the active pressure before the addition of the relaxant stimulus or agent, i.e. the baseline pressure. Changes in the trough-to-peak pressure produced by volume oscillations were used as an index of stiffness under dynamic conditions and include the wall elasticity and the viscous resistance of the wall to movement, making it a measure of wall impedance that shows how hard it is to inflate the bronchi.

Response to relaxant drugs, EFS and oscillation were analysed using one- or two-way ANOVA, or a paired t-test. Repeated measure ANOVA was used where appropriate. Values are presented as mean \pm SEM and $n$ represents the number of preparations and animals.

\section{RESULTS}

Active airway pressure (contraction) was recorded under both static and oscillatory conditions. In the absence of ASM activation, the trough-to-peak amplitudes of the pressure cycles produced by volume oscillation were $4.7 \pm 0.4 \mathrm{cmH}_{2} \mathrm{O}$ $(0.46 \pm 0.04 \mathrm{kPa})$ for tidal oscillation and $11.7 \pm 0.6 \mathrm{cmH}_{2} \mathrm{O}$ $(1.15 \pm 0.06 \mathrm{kPa})$ for twice tidal oscillation. In the presence of ASM activation with carbachol, the amplitudes of both cycling regimes increased to $21.6 \pm 2.9 \mathrm{cmH}_{2} \mathrm{O}(2.12 \pm 0.28 \mathrm{kPa})$ under tidal oscillation (fig. 1) and $48.2 \pm 5.6 \mathrm{cmH}_{2} \mathrm{O}(4.72 \pm 0.55 \mathrm{kPa})$ under twice tidal oscillation, indicating an increase in airway stiffness as well as concentration [12]. However, the active pressure produced by carbachol $\left(10^{-6} \mathrm{M}\right)$ in oscillating airways was substantially less than in the static condition and was reduced by $27.8 \pm 4.3 \%$ during tidal oscillation and $66.8 \pm 4.1 \%$ during twice tidal oscillation $(\mathrm{p}<0.001$; repeated measures oneway ANOVA; $\mathrm{n}=12$ ).

In atropinised airway preparations that were contracted with carbachol, trains of EFS produced relaxation (fig. 2). Previous studies suggested that the relaxant responses to EFS under the described conditions were neural in origin since they are abolished by the nerve blocker tetrodotoxin [9]. This was confirmed in the present study (data not shown). The neurotransmitter responsible for the relaxation was investigated in four preparations incubated in the nitric oxide synthase inhibitor L-NOARG $\left(10^{-4} \mathrm{M}\right)$ for $\sim 30 \mathrm{~min}$ under static conditions. L-NOARG largely abolished relaxation to EFS (95.4 $\pm 3.9 \%$ inhibition of response), without altering carbachol-induced contraction. Mean EFS responses recorded under the static and dynamic conditions are shown in figure 3. With the reduced active pressure in oscillating airways the

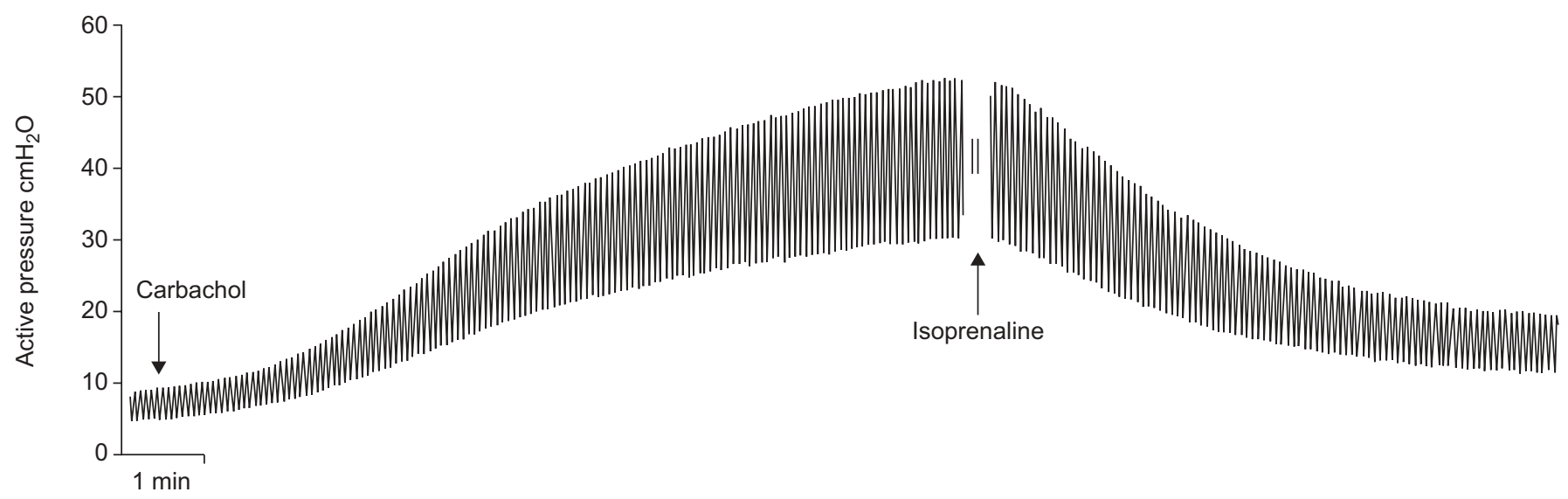

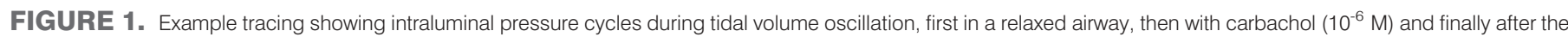

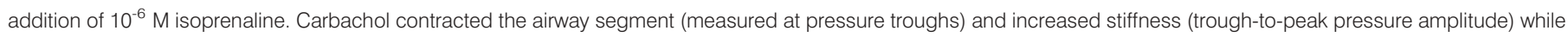
isoprenaline relaxed the airway reducing stiffness. 

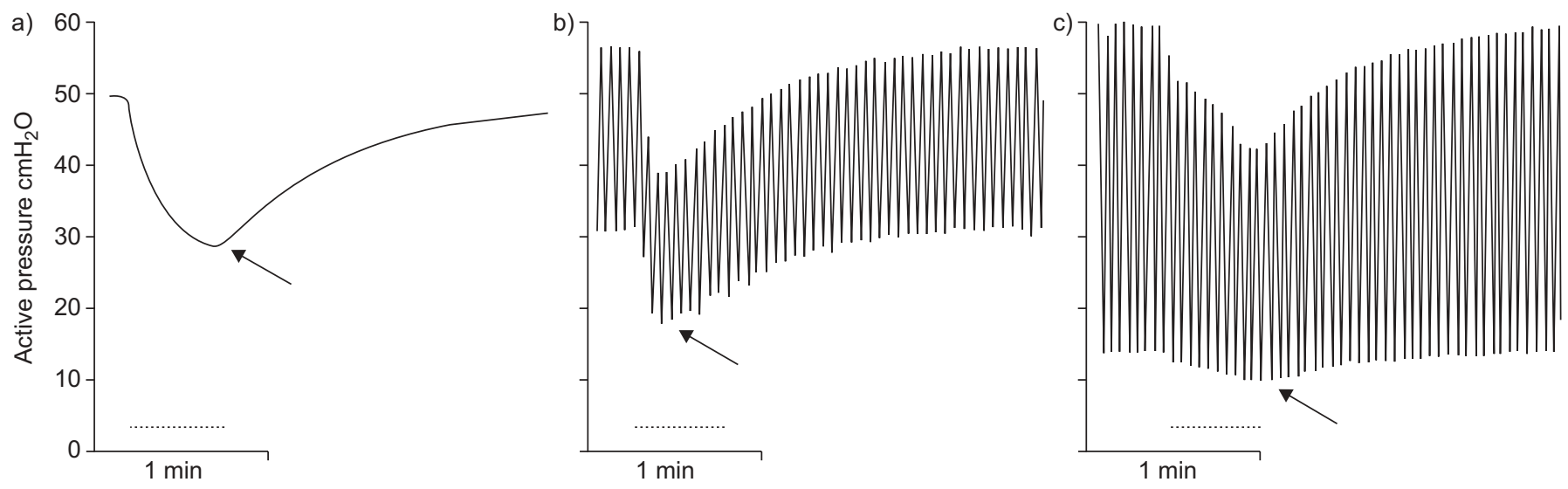

FIGURE 2. Sample intraluminal pressure traces $\left(\mathrm{cmH}_{2} \mathrm{O}\right)$ recorded in a pig isolated airway contracted with carbachol $\left(10^{-5} \mathrm{M}\right)$ under a) static and oscillating volume conditions, b) tidal and c) twice tidal. Volume oscillation produced cyclical pressures in the airway lumen, which were greater for twice tidal volume oscillations than tidal oscillation. Oscillation also reduced carbachol-induced active pressure in an amplitude-dependent manner, as recorded at isovolume points (i.e. the trough pressure under oscillating conditions). Electrical field stimulation ( $2 \mathrm{~ms}, 20 \mathrm{~Hz}, 299 \mathrm{~mA}$ applied for $30 \mathrm{~s} ; \cdots \cdot \cdots)$ produced a transient reduction in active pressure at isovolume points (arrows) under all conditions.
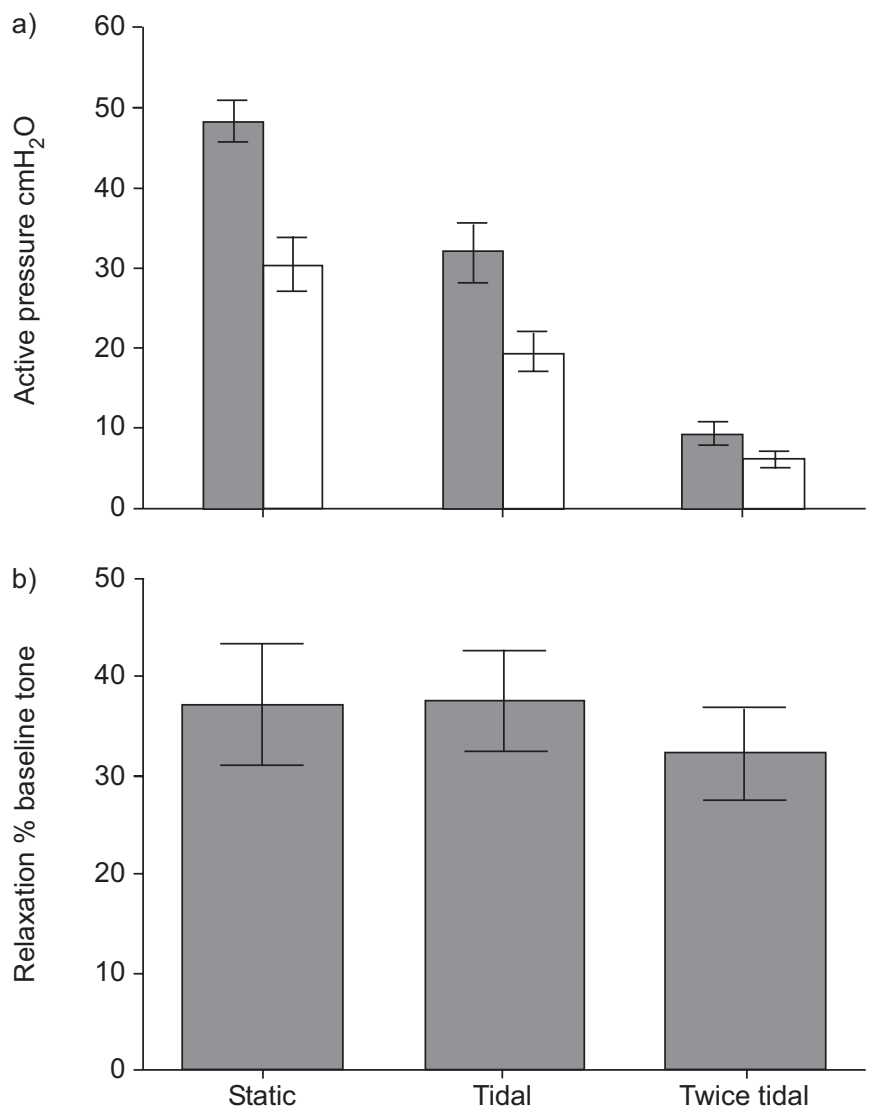

FIGURE 3. a) Relaxation of carbachol-induced tone $\left(\mathrm{cmH}_{2} \mathrm{O}\right)$ by electrical field stimulation (EFS; $\square$ ) in isolated airways under static, tidal and twice tidal oscillation. Data show the active pressure before $(\square)$ and after EFS ( $p<0.0001-0.01$; repeated measures two-way ANOVA). b) When response to EFS was normalised to baseline pressure (\% baseline tone) there was no significant difference ( $p>0.05$; matched one-way ANOVA; $n=6$ ) between the per cent response under static and oscillatory conditions. absolute decrease in pressure in response to EFS was also reduced. However, when normalised against baseline tone (active pressure) just before stimulation, the percentage of relaxation was similar under the different conditions $(37.1 \pm 6.0 \%, 37.4 \pm 5.1 \%$ and $32.2 \pm 4.4 \%$ for static, tidal and twice tidal, respectively; $\mathrm{p}>0.05$; repeated measures one-way ANOVA; $n=6)$.

Under static conditions both SNP and isoprenaline produced concentration-dependent relaxation (figs $4 a$ and 5a). Similarly, SNP and isoprenaline produced relaxation in airways subjected to oscillation in which active pressure was already reduced by oscillation. When normalised to the baseline tone before the addition of a drug, the isoprenaline-induced relaxations were similar under oscillating and static conditions (fig. 4b). Normalised relaxation responses to SNP, however, were enhanced by both oscillation parameters $(\mathrm{p}<0.001$; repeated measures two-way ANOVA; $n=6$; fig. 5b).

As previously noted, carbachol increased airway stiffness as well as active lumen pressure. In addition to reducing the airway active pressure as already discussed, each of the three inhibitory signals also reduced the stiffness, producing up to $\sim 80 \%$ reversal back to pre-carbachol stiffness levels with SNP and isoprenaline $(\mathrm{p}>0.0001-0.001$; repeated measures two-way ANOVA; $n=6)$ and 20-30\% reversal with EFS ( $p<0.05$; figs 1 and 6). There were no significant differences in the degree of reversal of airway stiffness under the different oscillating conditions ( $\mathrm{p}>0.05$; paired t-test; $\mathrm{n}=6$ ).

\section{DISCUSSION}

The present study compares the actions of a range of physiological and pharmacological stimuli under static and dynamic conditions likely to be encountered in vivo. ASM responses to contractile pharmacological agents are dependent on the prevailing dynamic conditions [1-4, 13]. However, little is known about the responsiveness of ASM or airways to other commonly used inhibitory pharmacological or physiological stimuli under dynamic conditions. The current findings 

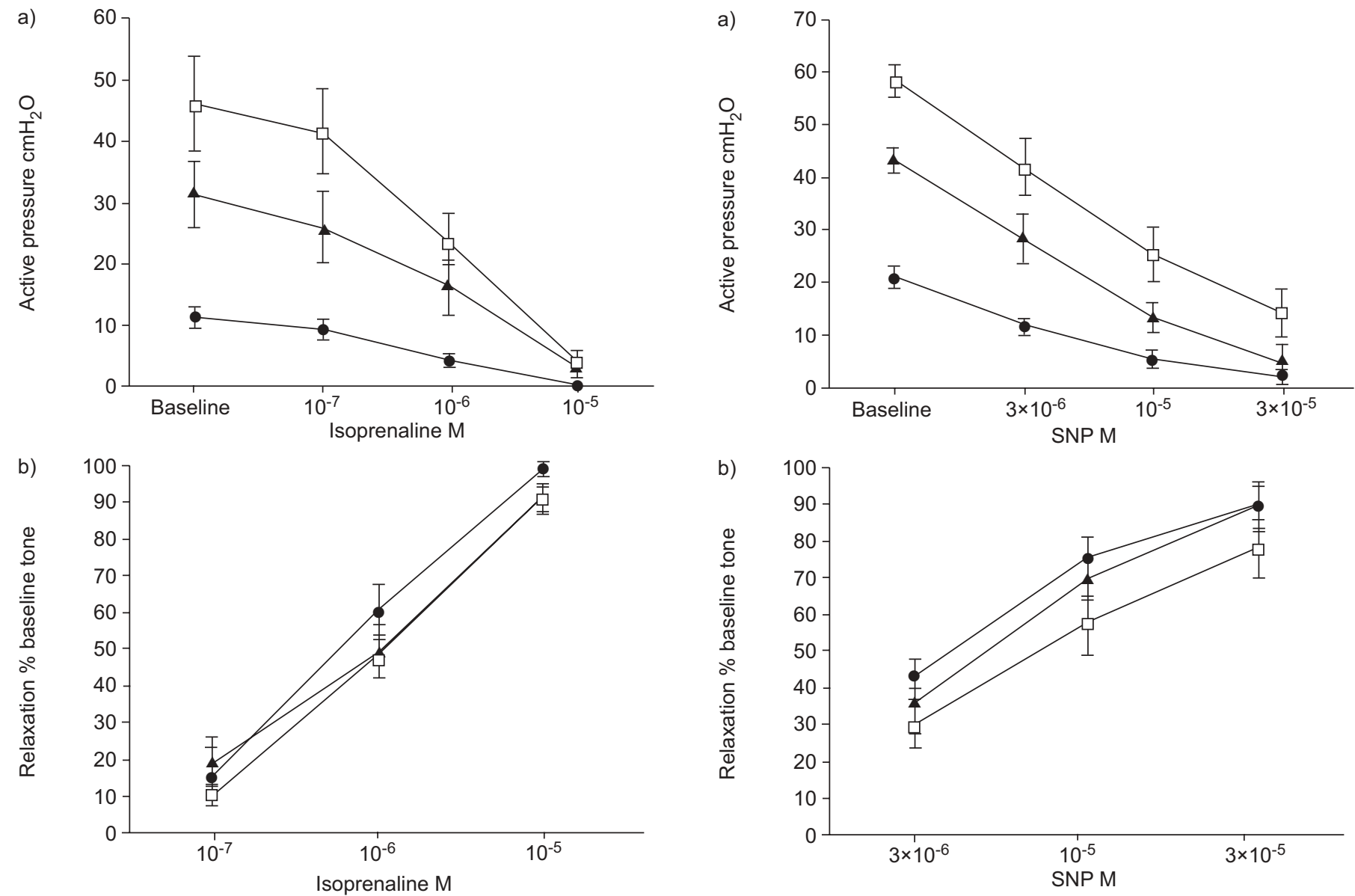

FIGURE 4. Concentration-response curves to isoprenaline $\left(10^{-7}, 10^{-6}\right.$ and $\left.10^{-5} \mathrm{M}\right)$ in isolated airways contracted with carbachol $\left(10^{-6} \mathrm{M}\right)$. a) Relaxation of carbachol-induced active pressure $\left(\mathrm{cmH}_{2} \mathrm{O}\right)$ by isoprenaline is shown under static $(\square)$ and oscillatory conditions: tidal $(\mathbf{\Lambda})$ and twice tidal $(\bullet)$. Isoprenaline and oscillation each caused relaxation $(p<0.0001-0.01$; repeated measures two-way ANOVA). b) The response to isoprenaline was normalised to baseline pressure (\% baseline tone) for each airway. There was no significant difference $(p>0.05$; repeated measures two-way ANOVA; $n=6$ ) between percentage relaxation under static and oscillatory conditions.

suggest that in a dynamic environment, ASM oscillation combines with neural and pharmacological relaxation and leads to major inhibition of ASM tone and, thus, airway narrowing. Additionally, the inhibitors reduce the stiffness of the airway wall, which in vivo may be an important component of bronchodilator efficacy.

The attenuation of ASM force by either single or continuing changes in muscle length is well documented [1-4, 13]. Thus, in vivo, it is likely that airway tone or calibre is regulated by an ongoing dynamic between fluctuating forces of breathing and the presence of either endogenous ASM contraction, or contraction evoked by pharmacological means, such as methacholine challenge. However, this is only one side of an equation, which may also include the activity or presence of inhibitory signals to ASM. These might include physiological activity from neural input, which is typically nitrergic, and $\beta$-adrenergic input either hormonally, or from drugs used in

FIGURE 5. Concentration-response curve to sodium nitroprusside (SNP $3 \times 10^{-6}, 10^{-5}$ and $\left.3 \times 10^{-5} \mathrm{M}\right)$ in isolated airways contracted with carbachol $\left(10^{-6} \mathrm{M}\right)$. a) Relaxation of carbachol-induced active pressure $\left(\mathrm{cmH}_{2} \mathrm{O}\right)$ by SNP is shown under static $(\square)$ and oscillatory conditions: tidal $(\mathbf{\Delta})$ and twice tidal $(\bullet)$. SNP and oscillation each caused relaxation $(p<0.001$; repeated measures two-way ANOVA). b) The response to SNP was normalised to baseline pressure (\% baseline tone) for each airway. The percentage response to SNP was significantly increased under each oscillating condition $(p<0.0001$; repeated measures two-way ANOVA; $n=6)$.

the management of obstructive airway disease. The present study provides information on airway relaxation under dynamic conditions. In keeping with previous studies in whole airway preparations [12, 13], cyclical oscillations to lumen volume suppressed active force or, as measured herein active lumen pressure. Two levels of volume oscillation were used in relaxed airways to produce pressure fluctuations corresponding to tidal breaths $\left(5 \mathrm{cmH}_{2} \mathrm{O}\right.$ amplitude) and a volume twice that amount which corresponds to deeper breathing between tidal breaths and sighs. All three inhibitory interventions (EFS, SNP and isoprenaline) produced further relaxation in addition to that produced by mechanical oscillation, so that overall airway tone was markedly reduced compared with stimulus-induced relaxations recorded under more conventional isometric protocols. For example, the combined effect of mid concentrations of SNP or of isoprenaline, coupled with tidal oscillation, reduced airway tone by $\sim 70 \%$, while when combined with twice tidal oscillation, tone was almost abolished. 

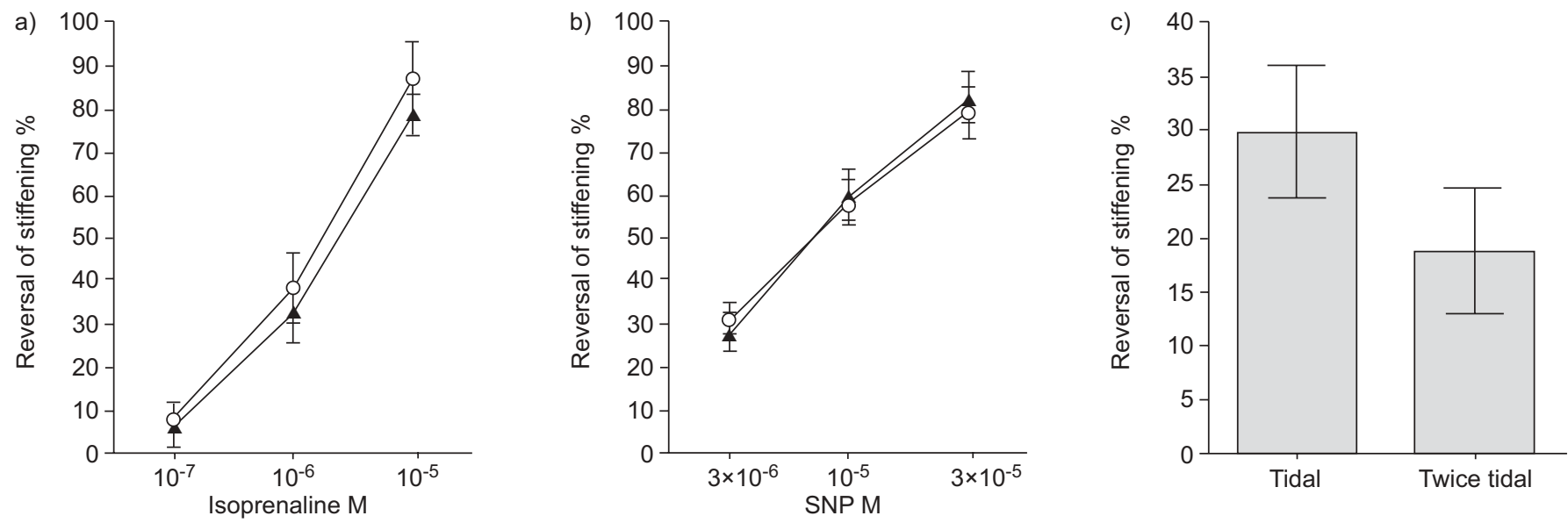

FIGURE 6. Reduction in airway stiffness by isoprenaline, sodium nitroprusside (SNP) and electrical field stimulation (EFS). Stiffness was assessed from the amplitude of trough-to-peak pressure oscillations during tidal $(\mathbf{\Lambda})$ and twice tidal $(O)$ volume oscillations. The reduction in airway stiffness to each relaxant is expressed as a percentage of the initial increase in stiffness produced by carbachol, referred to here as percentage reversal. a) The maximal concentration of isoprenaline $\left(10^{-5} \mathrm{M}\right)$ caused $\sim 85 \%$ reversal back to relaxed stiffness. There was no significant difference ( $p>0.05$; repeated measures two-way ANOVA) between the responses under tidal and twice tidal oscillation. $b$ ) The maximal concentration of SNP $\left(3 \times 10^{-5} \mathrm{M}\right)$ caused $\sim 80 \%$ reversal back to relaxed stiffness. There was no significant difference ( $p>0.05$; repeated measures two-way ANOVA) between the responses under tidal and twice tidal oscillation. c) EFS caused $20-30 \%$ reversal of stiffness back to relaxed stiffness ( $p<0.05)$. There was no significant difference $(p>0.05$; repeated measures two-way ANOVA; $n=6)$ between the responses under tidal and twice tidal oscillation.

Since there was less active tone in oscillating airways, the absolute magnitudes of evoked relaxations were less under dynamic conditions compared with static. Therefore, relaxation was normalised for baseline tone. In the case of EFS and isoprenaline, normalised relaxations were no different between static and either of the dynamic conditions. Thus the combined relaxant effect of two interventions (oscillation and evoked relaxation) was the same or similar to the multiple of the fractional changes to the two. EFS induced relaxation was blocked by L-NOARG, a blocker that has been used previously to identify nitric oxide-mediated neural relaxation $[8,9]$. The multiplicative model of response to EFS or isoprenaline and oscillation could indicate that the interventions act at separate, noninteracting sites, as previously suggested by GuMP et al. [4] for isoprenaline. EFS and isoprenaline act upstream of the contractile machinery, and as such the absence of interaction between oscillation and evoked relaxation is consistent with the molecular mechanisms thought to account for the effects of mechanical strain on ASM contraction [3,5].

However, findings with SNP differed from EFS and isoprenaline in that normalised responses to SNP were greater under the oscillating conditions and, importantly, greater than that predicted from a multiplicative model. While the differences between relaxation in static and oscillating conditions were not large (i.e. a $10-20 \%$ difference in normalised relaxation in figure $5 \mathrm{~b}$ amounts to only a few $\mathrm{cmH}_{2} \mathrm{O}$ ), the finding has a number of possible implications. First is the apparent conflict with EFS, where nerve mediated relaxation responses were unaffected by oscillation while relaxation to exogenously applied nitric oxide was enhanced. SNP is a nitric oxide donor and should, in large, part mimic the post-junctional component of EFS. Given the common post-junctional pathways for SNP and EFS, one could suggest that oscillation exerts some inhibitory pre-junctional effect on the release of the neurotransmitter, which then counters the increased post-junctional effects of oscillation. This possibility seems unlikely because any inhibitory effect of oscillation on transmitter release would have to exactly match the increased effects of nitric oxide on the ASM since EFS-induced responses were the same under static and oscillatory conditions. Secondly, the increased postjunctional effect of SNP combined with strain oscillation suggests that the two pathways may interact. Although SNP acts via cyclic guanosine monophosphate and therefore upstream of cross bridge kinetics and cytoskeletal proteins, some mechanistic interaction between SNP and ASM strain cannot be excluded. Lastly, a possible physiochemical explanation for the enhanced effects of SNP with oscillation can be offered. Contact with tissue in the physically stirred environment of the oscillating airway may have promoted the chemical reduction of SNP to release nitric oxide [14, 15], thereby, increasing the amount of relaxation produced. In that case the increased relaxation under oscillating conditions might be, in a sense, an artefact of the system rather than reflect a true signalling interaction.

By utilising a whole airway preparation the present authors were also able to identify a powerful softening of the contracted airway wall by all three inhibitory interventions. Contractile activation increases ASM stiffness [16], which increases the stiffness of the intact airway wall to a degree that far outweighs passive wall stiffness in the absence of ASM contraction [12]. The strong stiffening action of carbachol on the airway wall was also observed in the present study, as evidenced by the four-fold increase in trough-to-peak pressure in the presence of carbachol. SNP and isoprenaline reduced carbachol-induced stiffness in a concentration dependent manner so that at high concentrations, stiffness had almost returned to pre-carbachol levels. Under dynamic conditions, both the wall elasticity and the wall tissue resistance (viscosity/viscoelasticity) alter the pressure swing (trough-topeak) for a fixed volume change. An effect of bronchodilators 
and inhibitory neural stimulation on stiffness has not been previously reported at an airway level, although $\beta$-adrenergic activation is known to reduce stiffness at cellular level [17-19]. This newly described action of physiological and pharmacological inhibitors, at a more integrated level of organisation in activated airways, may have considerable significance to the maintenance of airway tone in vivo. The capacity of deep inspirations and tidal breathing to maintain the patency of airways in vivo is well established [20]. This effect is secondary to the ASM strain that in turn is produced by the transmural pressure and stress in the airway during breathing. Strain is critically subject to airway stiffness; the lesser the stiffness the greater the strain with breathing movements, the greater the reduction of ASM force and so the greater the likely bronchodilation [12]. Bronchodilator actions of $\beta$-adrenoreceptor agonists and neural stimulation are usually considered within the framework of their direct effects on ASM contraction. The present findings suggest a different concept, in which inhibitory neural and pharmacological signals also promote bronchodilation by reducing airway stiffness, maximising the strain-induced reduction in ASM force and so amplifying bronchodilation. As strain is considered to be one of the most powerful regulators of airway calibre [4], elastic and viscous properties of the airway in the presence of bronchodilator stimuli may be of major importance to their overall effects in reversing airway constriction.

The above paradigm might be more important in vivo, where, unlike in the present study, the airway length is free to extend as well as dilate. ASM is aligned approximately circumferentially around the airway, so that with ASM contraction and circumferential stiffening, airways may be more prone to lengthening on inspiration, as discussed by MAKsYM et al. [21]. An increased tendency of airways to lengthen during breathing could offset the amount of circumferential ASM strain and thus increase ASM force produced by the bronchoconstrictor stimulus. Should ASM strain fall below a critical value (1-2\%) then the relaxant effect of mechanical stain is potentially lost [4, 12]. As suggested previously, that by softening airways circumferentially bronchodilators might increase ASM strain, increasing relaxation can be developed further by proposing a shift in balance between airway length and diameter during inspiration, back towards airway dilation and away from lengthening. Bronchodilator efficacy could then be considered in a three-dimensional context of the relative circumferential and axial stiffness of the airway, the corresponding airway expansion produced by the stresses of respiration (i.e. dilation versus lengthening) and ASM force.

In conclusion, the present authors have shown that breathing movements combined with inhibitory innervation or pharmacological bronchodilators produces a powerful fall in airway smooth muscle contraction. The actions of airway smooth muscle stretch and relaxation through either the cyclic adenosine monophosphate or cyclic guanosine monophosphate pathways seem to be largely independent with little major effect of oscillation on inhibitory response once corrected for the lower level of tone. However, inhibitory agonists and nerves also reduced airway stiffness that in vivo may allow for greater airway smooth muscle stretch for a fixed change in transpulmonary pressure during tidal or deep inspiration breaths, adding to their bronchodilator efficacy.

\section{REFERENCES}

1 Shen X, Wu MF, Tepper RS, Gunst SJ. Pharmacological modulation of the mechanical response of airway smooth muscle to length oscillation. J Appl Physiol 1997; 83: 739-745.

2 Shen X, Tepper RS, Gunst SJ. Mechanisms for the mechanical response of airway smooth muscle to length oscillation. J Appl Physiol 1997; 83: 731-738.

3 Fredberg JJ, Inouye D, Miller B, et al. Airway smooth muscle, tidal stretches, and dynamically determined contractile state. Am J Respir Crit Care Med 1997; 156: 1752-1759.

4 Gump A, Haughney L, Fredberg J. Relaxation of activated airway smooth muscle: relative potency of isoproterenol vs. tidal stretch. J Appl Physiol 2001; 90: 2306-2310.

5 Gunst SJ, Tang DD, Opazo Saez A. Cytoskeletal remodeling of the airway smooth muscle cell: a mechanism for adaptation to mechanical forces in the lung. Respir Physiol Neurobiol 2003; 137: 151-168.

6 Fust A, Stephens NL. Relaxation of canine airway smooth muscle. Can J Physiol Pharmacol 2007; 85: 672-678.

7 Belvisi MG, Stretton D, Yacoub M, Barnes PJ. Nitric oxide is the endogenous neurotransmitter of bronchodilator nerves in humans. Eur I Pharmacol 1992; 210: 221-222.

8 Kannan MS, Johnson DE. Nitric oxide mediates the neural nonadrenergic, noncholinergic relaxation of pig tracheal smooth muscle. Am J Physiol 1992; 262: L511-L514.

9 Connellan DR, Mitchell HW. Transition of functional innervation in the developing porcine airway from nitrergic to catecholaminergic. Brit J Pharmacol 1998; 123: 712-718.

10 Barnes PJ. Endogenous inhibitory mechanisms in asthma. Am J Respir Crit Care Med 2000; 161: S176-S181.

11 Noble PB, Sharma A, McFawn PK, Mitchell HW. Airway narrowing in porcine bronchi with and without lung parenchyma. Eur Respir J 2005; 26: 804-811.

12 Noble PB, McFawn PK, Mitchell HW. Responsiveness of the isolated airway during simulated deep inspirations: effect of airway smooth muscle stiffness and strain. J Appl Physiol 2007; 103: 787-795.

13 Gunst SJ, Stropp JQ, Service J. Mechanical modulation of pressure-volume characteristics of contracted canine airways in vitro. J Appl Physiol 1990; 68: 2223-2229.

14 Rochelle LG, Kruszyna H, Kruszyna R, Barchowsky A, Wilcox DE, Smith RP. Bioactivation of nitroprusside by porcine endothelial cells. Toxicol Appl Pharmacol 1994; 128: 123-128.

15 Bates JN, Baker MT, Guerra R Jr, Harrison DG. Nitric oxide generation from nitroprusside by vascular tissue. Evidence that reduction of the nitroprusside anion and cyanide loss are required. Biochem Pharmacol 1991; 42: Suppl., S157-S165.

16 Stamenovic D, Suki B, Fabry B, Wang N, Fredberg JJ, Buy JE. Rheology of airway smooth muscle cells is associated with cytoskeletal contractile stress. J Appl Physiol 2004; 96: 1600-1605.

17 Fabry B, Maksym GN, Shore SA, et al. Selected contribution: time course and heterogeneity of contractile responses in cultured human airway smooth muscle cells. J Appl Physiol 2001; 91: 986-994. 
18 Ito S, Majumdar A, Kume $\mathrm{H}$, et al. Viscoelastic and dynamic nonlinear properties of airway smooth muscle tissue: roles of mechanical force and the cytoskeleton. Am J Physiol Lung Cell Mol Physiol 2006; 290: L1227-L1237.

19 Mehta D, Gunst SJ. Actin polymerization stimulated by contractile activation regulates force development in canine tracheal smooth muscle. J Physiol 1999; 519: 829-840.
20 Brusasco V, Pellegrino R. Complexity of factors modulating airway narrowing in vivo: relevance to assessment of airway hyperresponsiveness. J Appl Physiol 2003; 95: 1305-1313.

21 Maksym GN, Deng L, Fairbank NJ, Lall CA, Connolly SC. Beneficial and harmful effects of oscillatory mechanical strain on airway smooth muscle. Can J Physiol Pharmacol 2005; 83: 913-922. 